Rapid Reviews COVID-19

\title{
Reviews of "Evidence for increased breakthrough rates of SARS-CoV-2 variants of concern in BNT162b2 mRNA vaccinated individuals"
}

Art Reingold ${ }^{1}$, Konstantin Chumakov ${ }^{2}$, Shivakumar Narayanan ${ }^{3}$, Shyam Kottiliil ${ }^{3}$, Joel V. Chua ${ }^{3}$

${ }^{1}$ UC Berkeley, ${ }^{2}$ Office of Vaccines Research and Review, U.S. Food and Drug Administration, ${ }^{3}$ Institute of Human Virology, University of Maryland School of Medicine, Baltimore, MD, USA.

Published on: Apr 15, 2021

DOI: $10.1162 / 2 \mathrm{e} 3983 f 5 . c 44 f 160 \mathrm{c}$

License: Creative Commons Attribution 4.0 International License (CC-BY 4.0). 


\section{RR:C19 Editorial Team Comment}

As vaccine shots go into arms around the world and SARS-CoV-2 variants continue to spread, people want to know to what extent COVID-19 vaccines protect against infection from previously identified Variants of Concern (VOCs). Early studies measuring the neutralizing ability of antibodies isolated from vaccinated individuals offered mixed results against different VOCs, and these reports also did not take into account other known in vivo mechanisms of viral clearance. This preprint seeks to address these outstanding questions.

In this study, authors examined clinical data from individuals vaccinated with the BNT-162b2 mRNA vaccine (BioNTech/Pfizer) who had a positive PCR test between 14 days after their first dose and 7 days after their second dose. The study found that partially and fully vaccinated individuals, when compared to demographically- and temporally-matched unvaccinated individuals, were more likely to be infected with VOCs than with the wildtype, or "original," strain of SARS-CoV-2. Partially vaccinated individuals were more likely than unvaccinated individuals to be infected with the B.1.1.7 strain ("U.K. variant") than with the wildtype strain. Fully vaccinated individuals were more likely than unvaccinated individuals to be infected with the B.1.351 strain ("South African variant") than with the wildtype strain.

These results, if verified, suggest that the B.1.1.7/U.K. variant is more effective at breaking through partial vaccination than the wildtype strain; and that the B.1.351/S.A. variant is more effective at breaking through full vaccination than the wildtype strain.

Rapid Reviews: COVID-19 consulted experts to review the strength of the data and claims, which can be found below. While this study must be understood in the context of other studies focused on vaccine efficacy overall against all strains of SARS-CoV-2, the conclusions of this study may have important public health implications, particularly for delayed vaccine dosing.

(RR:C19 Editorial Team Comment / Written by Christine Davis (MIT) and Michael Cronce (UC Berkeley)

Summary of Reviews: This preprint investigates SARS-CoV-2 variant breakthrough rates and finds VOCs more prevalent in COVID19+ vaccinees relative to the unvaccinated. Reviewers deem claims compelling, but warn findings do not concern disease severity and larger follow-up studies are needed.

To read the original manuscript, click the link above. To read the peer reviews, click the links below.

\section{Reviewer 1 (Art Reingold) |}

\section{Reviewer 2 (Konstantin Chumakov)}


Reviewer 3 (Shivakumar Narayanan, Shyam Kottilil, Joel V. Chua) | $\square\left[\begin{array}{c}\square \\ \square\end{array}\right.$

RR:C19 Strength of Evidence Scale Key.

$$
\begin{aligned}
& \text { प प्र०प = Misleading } \\
& \text { प्र } \square \square \square=\text { Not Informative } \\
& \text { प्रा } \square \square=\text { Potentially Informative }
\end{aligned}
$$

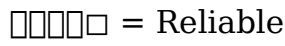

$$
\begin{aligned}
& \text { प्राप्र = Strong }
\end{aligned}
$$

To read the reviews, click the links below. 